\title{
Diffraction and central exclusive production at ATLAS
}

\author{
Marek Taševský, on behalf of the ATLAS collaboration \\ Institute of Physics, Academy of Sciences of the Czech republic, Na Slovance 2, 18221 Prague, \\ Czech republic
}

\begin{abstract}
The diffractive physics program for the ATLAS experiment with an emphasis on the central exclusive production is discussed. The key point in this discussion is the need for an unambiguous experimental definition of diffractive signature which would be acceptable and reproducible by theorists. Recent ATLAS results from samples enhanced in diffraction contribution underline this need.
\end{abstract}

Keywords: Diffraction, Central exclusive production, Higgs boson, Rapidity gap

PACS: $13.85 . \mathrm{Hd}$

\section{INTRODUCTION}

The central exclusive production (CEP) of new particles has received a great deal of attention in recent years (see [1] and references therein). The process is defined as $p p \rightarrow p \oplus \phi \oplus p$ and all of the energy lost by the protons during the interaction (a few per cent) is used in the production of the central system, $\phi$. The final state therefore consists of a centrally produced system coming from a hard subprocess, two very forward protons and no other activity. The ' $\oplus$ ' sign denotes the regions devoid of activity, often called rapidity gaps. In Double Pomeron Exchange (DPE), the central system contains remnants from the diffractive exchange in addition to the hard subprocess. More details on the diffraction physics program of the ATLAS experiment can be found e.g. in $[2,3]$.

\section{WITHOUT PROTON TAGGING}

At low luminosities, when there are on average few proton-proton interactions per bunch crossing, the diffractive processes can be detected using rapidity gaps. A possible L1 trigger would be based on vetoing activity on either side of the interaction point (IP) and requiring activity in the central detector with energy over a certain threshold. The gap may span the region from the forward calorimeters of the ATLAS detector (FCAL) [4] over the Minimum Bias Trigger Scintillator (MBTS), the luminosity LUCID detector [5] up to the ZDC detector [6]. Measurements suitable for analyses of the early LHC data are ratios of the cross-section for $\mathrm{X}+$ gaps to the one for $\mathrm{X}$ (incl.), where $\mathrm{X}$ may be $\mathrm{W}, \mathrm{Z}$, dijet, heavy quark and dilepton, and $\mathrm{X}$ (incl.) means measuring $\mathrm{X}$ inclusively. In these measurements, many sources of systematic uncertainties cancel, particularly the one related to the luminosity. They also serve as valuable checks of different components of the formalism used to predict the CEP cross section (e.g. by the KMR group [7]). The 
soft survival probability, $S^{2}$ (probability that additional soft secondaries will not populate the gaps), can be studied in electroweak processes $\mathrm{W} / \mathrm{Z}+$ gaps. The generalized gluon distribution, $f_{g}$, can be probed in exclusive $\Upsilon$ production. The higher-order QCD effects, especially Sudakov-like factors and also a possible role of the enhanced absorptive corrections can be studied in exclusive two- or three-jet events.

\section{Jet production in CEP, DPE and SD}

First CEP dijet candidates in hadron-hadron collisions and a reasonable agreement with theoretical (KMR) predictions, although known with a factor of three uncertainty, were observed by CDF [8]. The motivation for a CEP analysis is to reduce this uncertainty and to constrain existing models and $f_{g}$. Due to the $J_{z}=0, C$-even, $P$-even selection rule applicable to all CEP processes, quark jet production is suppressed and the CEP candidates can be recognized thanks to a reduced ratio of $b$-jets to all jets when compared to other types of events. The main backgrounds, dijets in non-diffractive (ND), single-diffractive (SD) and DPE processes, may be efficiently reduced by requiring simultaneously an MBTS veto, rapidity gap at least on one side, a low number of tracks outside the dijet system and $R_{j j}$ (ratio of the dijet mass to the total mass) close to 1 .

The jet production in DPE (SD) can be measured by requiring central jets and rapidity gaps on both sides (one side) of the IP in the FCAL/LUCID/ZDC detectors. The rapidity gaps may be spoiled by particles from the Pomeron remnants. By measuring the ratio $\sigma\left(S D_{j j}\right) / \sigma\left(N D_{j j}\right)$ and knowing the proton PDFs from precise HERA measurements, one can get information on the diffractive PDFs (dPDFs) and $S^{2}$. At the Tevatron, this measurement revealed a strong factorization breaking [9] when compared to dPDFs from HERA. This is usually explained by multiple interactions and absorptions and leads to $S^{2} \approx 0.1$. By varying the gap size in a measurement of the ratio $\sigma\left(D P E_{j j}\right) / \sigma\left(N D_{j j}\right)$, one is able to study the effects of Sudakov suppression and enhanced absorption.

\section{First measurement with diffractive enhanced minimum bias events}

According to current models, the diffractive component to the inelastic interactions contributes typically 20-30\% at $\sqrt{s}=7 \mathrm{TeV}$. Uncertainties in predicting the behavior of diffractive events at LHC energies make it difficult to measure and model the ND portion and lead to uncertainties of $20 \%$ in the luminosity determination based on counting inelastic collisions [10].

In this study [11], the inclusive sample (any side) is formed by events with at least one good track with $p_{T}>0.5 \mathrm{GeV}$ and $|\eta|<2.5$. The diffractive enhanced sample is formed from the inclusive sample by requiring in addition hits on exactly one side of the MBTS. The former consists of 12-20\%, while the latter contains $85-98 \%$ diffractive processes, depending on which $\mathrm{MC}$ model is used. The results are not corrected for detector effects.

In Fig. 1 left, the ratio of single-sided to any-sided events, $R_{s s}$, is plotted. The measured value is $[4.52 \pm 0.02$ (stat.) \pm 0.61 (syst.) $] \%$. The MC predictions are shown as a function of the diffractive contribution to the total inelastic cross section. In this comparison the ratio of SD to DD contribution is kept fixed. PYTHIA6 [12] and PYTHIA8 [13] 
(with the diffractive contribution of about $30 \%$ ) describe the data well, while PHOJET [14] would need to increase it from $20 \%$ to $30 \%$.
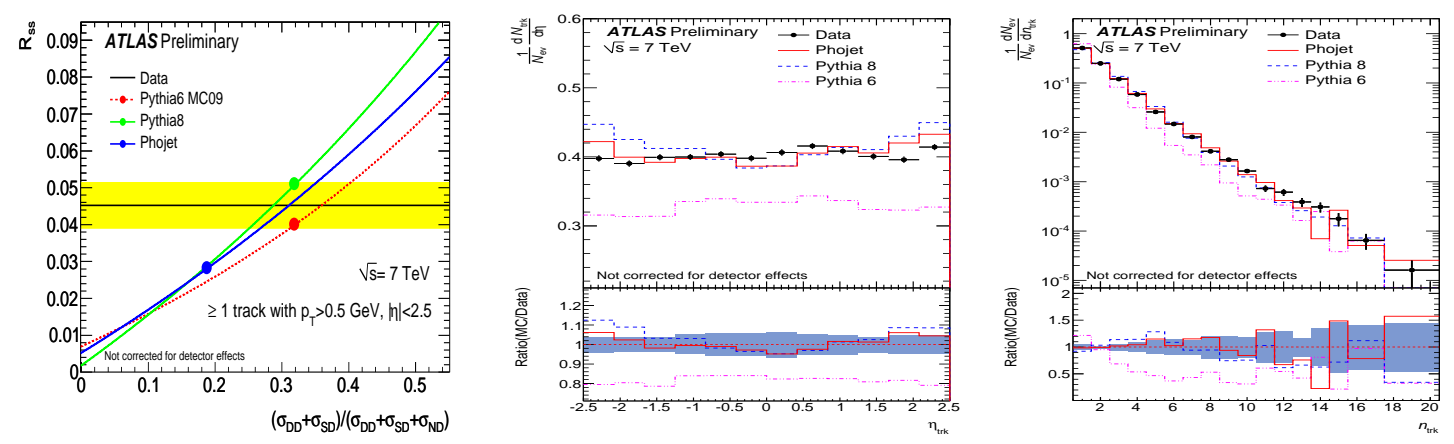

FIGURE 1. Left: The ratio $R_{s s}$ (defined in the text) compared to PYTHIA6 (red dotted), PYTHIA8 (green) and PHOJET (blue); The track distributions for $\eta$ (middle) and multiplicity (right) for the singlesided MBTS requirement, the data shown by points, PHOJET by full, PYTHIA8 by dashed and PYTHIA6 by dashed-dotted lines. The bottom plots show the ratio of MC to data, the shaded band indicates the quadratic sum of the statistical and systematic uncertainty.

Figure 1 shows the track $\eta$ (middle) and multiplicity $n_{\text {trk }}$ (right) distributions for the single-sided events. The $\eta$ distribution in the data is rather flat and the number of tracks per unit of $\eta$ is about 0.4 . The $n_{\text {trk }}$ distribution is much steeper than that measured in the inclusive sample [15]. Data are well modeled by PHOJET and PYTHIA8 in overall normalization, while PYTHIA6 underestimates the data (by about $30 \%$ in $\eta$ distribution), apart from the $n_{t r k}$ tail where the ND contribution is the most important.

\section{First measurement of dijet production with a jet veto}

In a recent study [16], the effects of QCD radiation are investigated in the rapidity interval between two selected jets. Possible radiation is restricted by a third-jet veto. An inclusive sample is formed by requiring single-jet L1 triggers and by selecting boundary jets with $p_{T_{1,2}}>30 \mathrm{GeV}$ and $\bar{p}_{T}=\left(p_{T 1}+p_{T 2}\right) / 2>60 \mathrm{GeV}$. The gap sample is formed from the inclusive sample by requiring in addition that there are no jets with $p_{T}>Q_{0}=$ $30 \mathrm{GeV}$ between the two boundary jets. The selection of the boundary jets is done in two ways: either two jets with the highest $p_{T}$ are required (selection $\mathrm{A}$ ), where one can study the effects of wide-angle soft-gluon radiation in the limit $\bar{p}_{T} \gg Q_{0}$. Or the most backward and most forward jet leading to a large jet rapidity separation, $\Delta y$, are required (selection B), where BFKL-like dynamics are expected to become important. Color singlet exchange can (in principle) be studied if both limits are satisfied at the same time, i.e. for large $\Delta y$ and $\bar{p}_{T} / Q_{0}$.

Figure 2 (left) shows the $p_{T}$ distribution of the third jet which exhibits a remarkable difference between the selections A and B. The gap fraction (defined as a ratio of events in the gap sample to that of the inclusive sample) corrected for detector effects is shown in Fig. 2 (middle and right) for the selection A (the selection B gives similar results). A reduction of the gap fraction with increasing $\bar{p}_{T}$ or $\Delta y$ is observed as well as a good description of the data by PYTHIA. More data will allow to enlarge the $\Delta y$ and $\bar{p}_{T}$ ranges and thus to explore in more detail the radiation pattern inside the rapidity gap. 

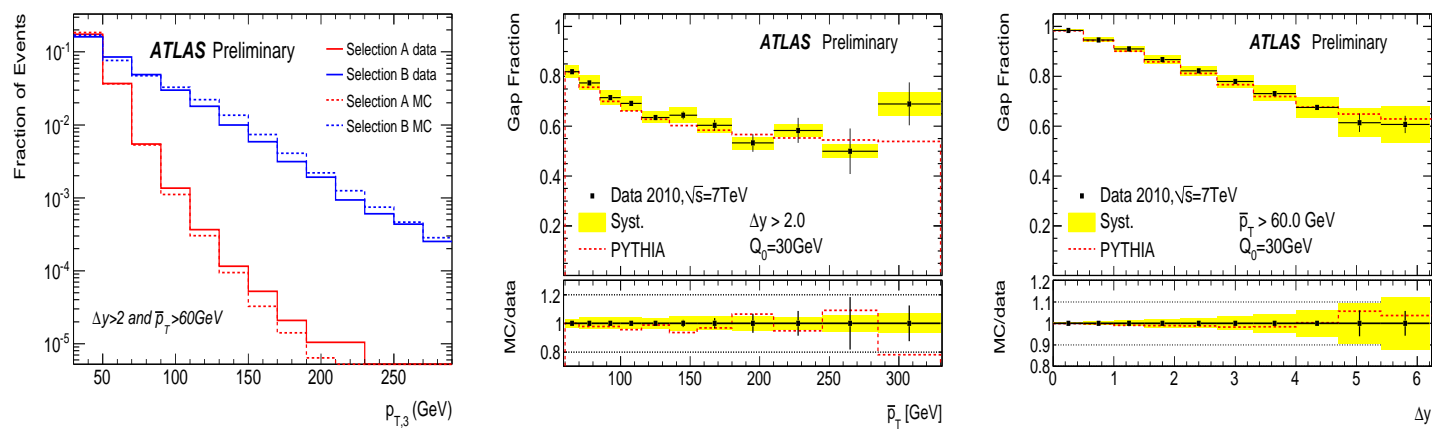

FIGURE 2. Left: $p_{T}$ distribution of the leading jet in the rapidity interval between the boundary jets; Gap fraction as a function of $\bar{p}_{T}$ (middle) and $\Delta y$ (right).

\section{WITH PROTON TAGGING}

The forward proton tagging will provide an exceptionally clean environment to search for new phenomena at the LHC and to identify their nature. A rich physics program can be pursued using proposed forward proton detectors in the AFP (ATLAS Forward Proton) project [17], see e.g. [1, 18]. It includes QCD, QED, EW, photon-induced and discovery physics. Of particular interest is the CEP of a SM (or MSSM) Higgs boson whose detection possibilities were intensively studied for various decay channels and mass regions (see an overview and references e.g. in [18]). The photon-induced interactions, a very rich physics chapter, are discussed elsewhere [19].

\section{ACKNOWLEDGMENTS}

Supported by the project AV0-Z10100502 of the Academy of Sciences of the Czech republic and project LC527 of the Ministry of Education of the Czech republic.

\section{REFERENCES}

1. FP420 R\&D Collab., J. Inst. 4 (2009) T10001. arXiv:0806.0302 [hep-ex].

2. M. Taševský, Nucl. Phys. Proc. Suppl. 179-180 (2008) 187; ATL-PHYS-CONF-2008-019.

3. C. Royon, arXiv:1008.3207 [hep-ex].

4. ATLAS Collab., J. Inst. 3 (2008) S08003.

5. ATLAS Collab., CERN-LHCC-2004-010 (2004).

6. ATLAS Collab., CERN-LHCC-2007-001 (2007); S. White, these proceedings.

7. V.A. Khoze, A. D. Martin and M. G. Ryskin, Eur. Phys. J. C23 (2002) 311; arXiv:0802.0177[hep-ph].

8. CDF Collab., Phys. Rev. D77 (2008) 052004.

9. CDF Collab., Phys. Rev. Lett. 84 (2000) 5043.

10. ATLAS Collab., ATL-CONF-2010-060, http: / / cdsweb. cern. ch/record/1281333.

11. ATLAS Collab., ATL-CONF-2010-048, http: / / cdsweb. cern . ch/record/1281302.

12. T. Sjöstrand, S. Mrenna and P. Skands, JHEP 05 (2006) 026.

13. T. Sjöstrand, S. Mrenna and P. Skands, Comput. Phys. Comm. 178 (2008) 852.

14. R. Engel, Z. Phys. C 66 (1995) 203.

15. ATLAS Collab., ATL-CONF-2010-024, http: / / cdsweb. cern. ch/record/1277656.

16. ATLAS Collab., ATL-CONF-2010-085, http: / / cdsweb. cern. ch/record/1298856.

17. ATLAS Collab., http: //www-hep.uta. edu/ brandta/ATLAS/AFP /AFP . html.

18. M. Taševský, arXiv:0910.5205 [hep-ph].

19. M. Albrow, these proceedings. 